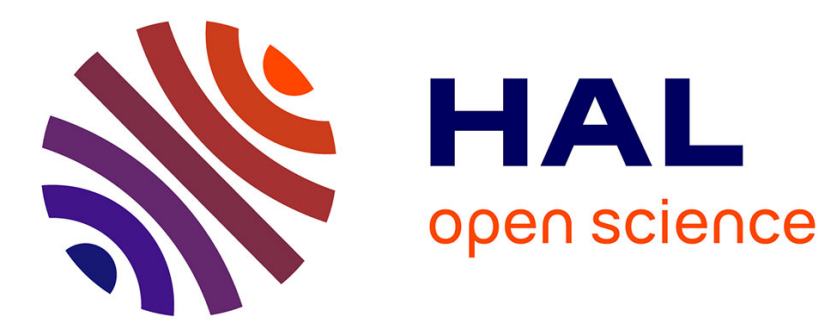

\title{
Comparison Between the Elasticity of Polyacrylamide and Polyacrylic Gels
}

Ludovic Benguigui

\section{To cite this version:}

Ludovic Benguigui. Comparison Between the Elasticity of Polyacrylamide and Polyacrylic Gels. Journal de Physique II, 1995, 5 (3), pp.437-443. 10.1051/jp2:1995132 . jpa-00248171

\section{HAL Id: jpa-00248171 https://hal.science/jpa-00248171}

Submitted on 1 Jan 1995

HAL is a multi-disciplinary open access archive for the deposit and dissemination of scientific research documents, whether they are published or not. The documents may come from teaching and research institutions in France or abroad, or from public or private research centers.
L'archive ouverte pluridisciplinaire HAL, est destinée au dépôt et à la diffusion de documents scientifiques de niveau recherche, publiés ou non, émanant des établissements d'enseignement et de recherche français ou étrangers, des laboratoires publics ou privés. 
Classification

Physics Abstracts

$62.20 \mathrm{D}-82.70 \mathrm{G}$

\title{
Comparison Between the Elasticity of Polyacrylamide and Polyacrylic Gels
}

\section{Benguigui}

Solid State Institute and Physics Department, Technion-Israel Institute of Technology, Haifa 32000 , Israel

(Received 25 March 1994, received in final form 7 November 1994, accepted 16 November 1994)

\begin{abstract}
We measured the Young modulus $E$ of polyacrylamide and polyacrylic gels as a function of the crosslink and polymer concentrations We found that the variation of $E$ with these two parameters is completely different for the two kinds of gels. For the polyacrylic gels, $E$ is an increasing function of the crosslink and polymer concentrations. However, in the case of the polyacrylamide gels, $E$ increases with polymer concentration but not always with the crosslink concentration. For low polymer concentrations, $E$ may saturate or exhibit a maximum when increasing the crosslink concentration. We proposed qualitative interpretations to explain this different behavior.
\end{abstract}

\section{Introduction}

The physical properties of gels and in particular therr elastic properties were and are still the subject of intense investigations [1]. In this paper we focus our attention on the elastic behavior of two different gels: polyacrylamide and polyacrylic gels. It is already known $[2,3]$ that the elastic modulus $G$ as a function of the crosslink content behaves differently for the two gels but it does seem that an extensive study of their elastic properties were made. The elasticity of these gels are very often presented among other properties and are not investigated in detail. In particular, the dependence of $G$ with the polymer concentration is not very well known. The purpose of this paper is to investigate the dependence of $G$ with the two basic parameters which control it: the crosslink concentration $c$ and the polymer concentration $p$.

These two kinds of gels are examples of two basic network defects: inhomogeneities (polyacrylamide gels) and entanglements (polyacrylic gels). Recently, these two types of defects have been studied theoretically by Vilgis [4] and as we shall show, our conclusions are not always in accordance with his theoretical predictions.

We recall two important points in the theory of the elastic modulus of a gel. First is the coefficient, $C_{0}$ which appears in the simple elastic modulus formula:

$$
G_{1}=C_{0} c k T
$$


In (1), $k$ is the Boltzman constant and $T$ the absolute temperature. The basic assumption in (1) is that there is no interaction between chains. There is a large amount of work devoted to the determination of $C_{0}$ [5]. For a functionality of four (as in our case) $C_{0}$ varies between $1 / 2$ and $1 / 4$. However, the important point is that $G_{1}$ has a linear dependence with $c$ and is independent of $p$ In (1), it is understood that $c$ is the effective crosslink density, which is not necessarily equal to the total crosslink density since dangling ends and loops do not contribute to the elastic modulus. The second point is the interaction between the chains which can be seen as closely packed blobs [6]. The influence of the chain interaction on the elastic modulus is a difficult problem. In general, the elastic modulus is written as the sum of two contributions $G=G_{1}+G_{2}$, where $G_{1}$ is given by (1) and $G_{2}$ is the new contribution. The concept of entanglements [4] is largely used and has the advantage of giving a simple physical picture of the chain interaction. Vilgis [4] proposed recently a method to calculate the elastic modulus as as function of $c$ and $p: G$ is no longer a linear function of $c$ as in (1), but remains an increasing function of $c$.

It is well-known that polymer gels are in general inhomogeneous $[4,7-10]$. The accepted picture is the existence of static inhomogeneities in which there are fluctuations in the crosslink molecules densities, with appearance of regions rich and others poor in crosslink molecules. In the case of the polyacrylamide gels, this picture is largely accepted as conclusion from different experimental results $[3,11-13]$. The recent results of Cohen et al. [3] are very interesting. They analyzed their SAXS by means of a simplified structure of the gel seen as a matrix with spherical inclusions rich in crosslink molecules. The radius of the inclusions is the mean size of the clusters with high crosslink molecule content. We mention that Weiss et al. [12] interpreted the results of permeation experiments using the same model.

\section{Experiments and Results}

We measured the Young modulus, which is equal to three times the shear modulus, varying the two important quantities. the crosslink concentration $c$ and the polymer concentration $p$.

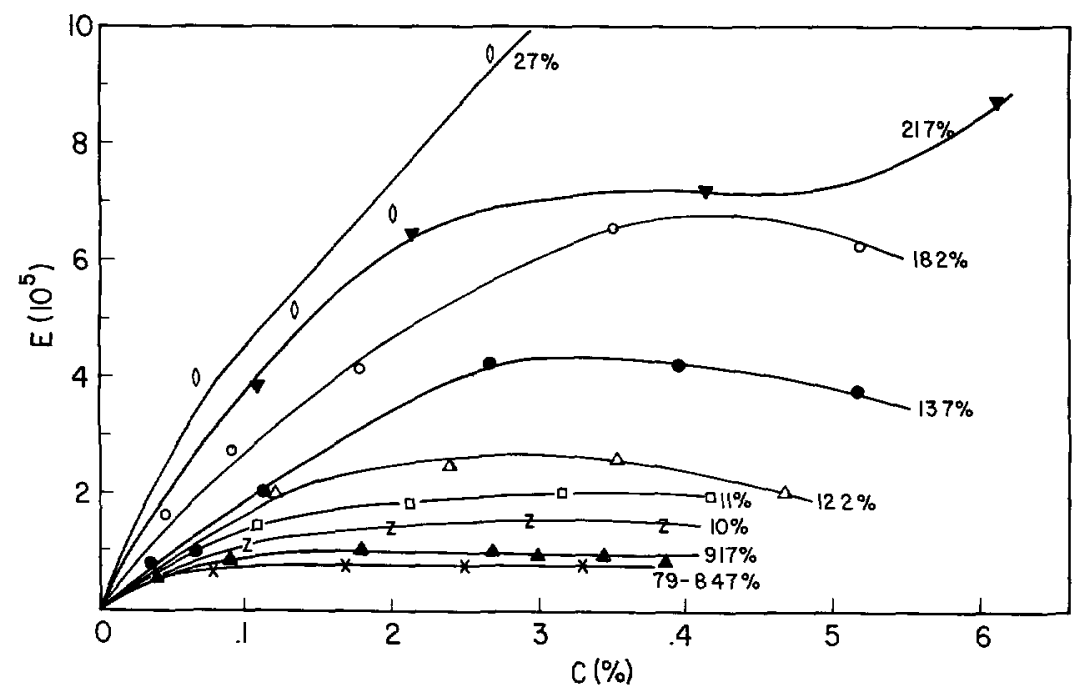

Fig. 1. - Young modulus of the polyacrylamide gels as a function of the crosslink concentration for different polymer concentrations. 


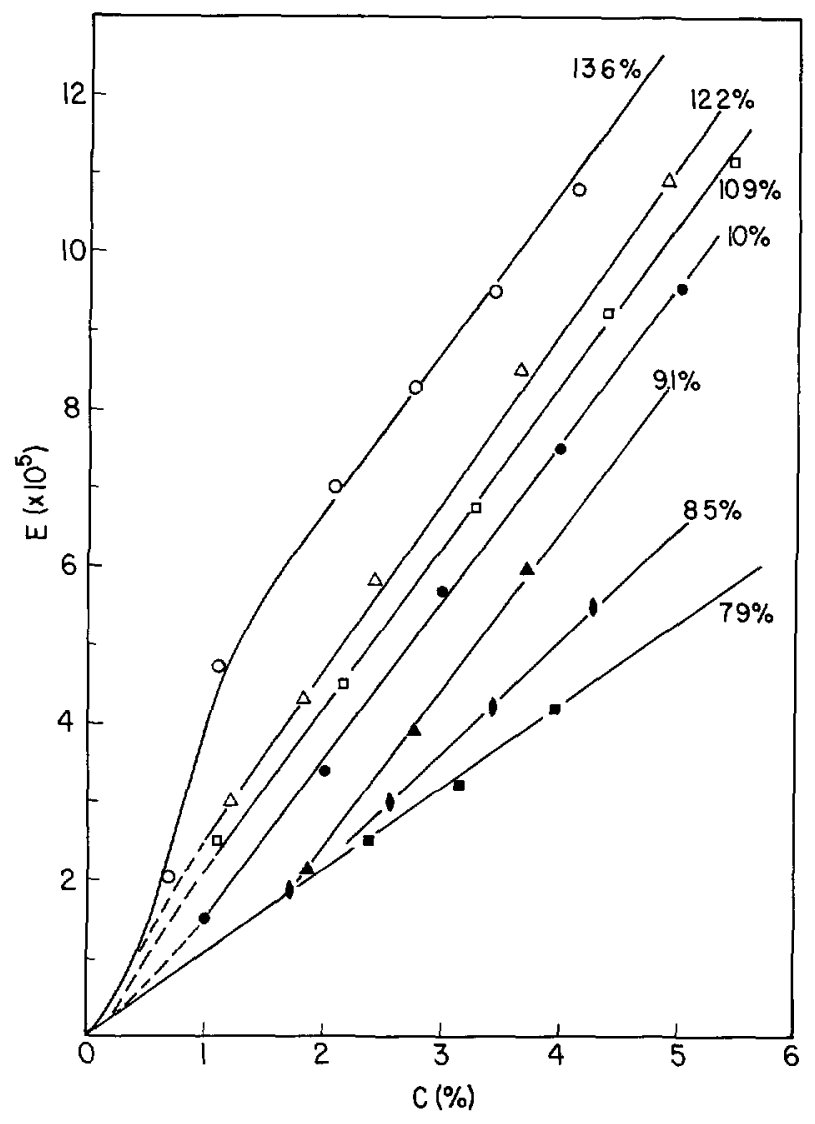

Fig, 2. - As in Figure 1 for polyacrylic gels

Both kinds of gel were prepared following essentially the same method with small differences. A given quantity of momomers (acrylic acid or acrylamide) was put in distilled water with the chosen amount of the tetrafunctional agent as crosslink ( $N, N^{\prime}$ - methylene bis acrylamide) with initiators. For the polyacrylic gels we took ammonium persulfate only, while for the polyacrylamide gels we took ammonium persulfate and tetramethylethylene (TEMED) [7]. In the case of polyacrylic acid, nitrogen was bubbled in the solution to remove oxygen which could inhibit the reactions. The polyacrylamide gels are ready in about $30 \mathrm{~min}$. at ambient temperature, but the polyacrylic gels need to be heated at $70^{\circ} \mathrm{C}$ for $8 \mathrm{~h}$. The samples have a cylindric shape (diameter $2.3 \mathrm{~cm}$ and length $3 \mathrm{~cm}$ ). In the preparation of the polyacrylic gels, we followed very closely the procedure indicated by the authors of reference [2]. With no addition of salt $(\mathrm{NaOH})$ or acid $(\mathrm{HCl})$, they give a degree of dissociation of $10^{-2}-5 \times 10^{-3}$. From their measurements of the elastic modulus as a function of $\alpha$ (up to $\alpha=0.6$ ), one can conclude that our gels are practically neutral.

The measurements were made on the samples as they were prepared with a home built apparatus. We impose a given deformation (compression) by moving a metallic cylinder by means of an eccentric wheel. The frequency of rotation is $0.3 \mathrm{~Hz}$, in the range of frequency for which the Young modulus is independent of the frequency. The force is measured from the signal given by five piezoelectric elements put in serie below the sample. We choose the linear 
regime of the curve stress-strain to determine the Young modulus.

In Figures 1 and 2, we show the curves $E(c)$ for different polymer concentrations. The concentrations in polymer $p$ and in crosslink $c$ are given in percentage of the solution (monomer + solvant), in volume: $p=10 \%$ means $10 \%$ of polymer and $90 \%$ of water in the solution. One sees immediately the difference between the two ensembles of curves and in each case one can distinguish several regimes. For the polyacryalamide gels one has four regimes: a) for small values of $c(c<0.1 \%)$ and whatever the value of $p, E$ increases with $c$. b) for larger value of $c$ and $p<10 \%, E$ saturates and becomes independent of $c$. c) for large $c$ and intermediate values of $p(10 \%<p<18 \%) E$ exhibits a maximum. d) for large $c$ and large $p(p>18 \%) E$ increases with $c$ sometimes with an inflexion point. The maximum in $E$ was already mentioned $[3,13]$ but it seems the different regimes of the Figure 1 were not observed before.

The Young modulus of the polyacrylic gels behaves very differently. It is always an increasing function of $c$ and $p$. For low value of $p$ (about $8 \%$ ), $E$ increases linearly with $c$ and again for the largest value of $p$. Between $p=8 \%$ and $p=14 \%$ there is a gradual passage from the behavior at low $p$ to that at larger $p$.

\section{Interpretation and Discussion}

The problem we want to address is the dramatic difference in the behavior of the two kinds of gel. The two kinds of gel were prepared following the same procedure. At the beginning the monomers are put in the solution with the crosslink. The gel is formed as a consequence of two reactions: the monomer-monomer (MM) reaction creating the polymer chains and the crosslink-monomer (CM) reaction giving the network. These two reactions do not take place at the same rate. We shall assume that the rates of the two reactions are different. For the polyacrylamide gels the rate of the CM reaction is much larger than that of the MM reaction. It follows that at first, units of one crosslink molecule and four monomers are formed and only after the chains are made. This process will create regions rich in crosslink agent and others with a lower density. The assumption regarding the different rates of the $\mathrm{MM}$ and $\mathrm{CM}$ reactions is confirmed in reference [13]. In the case of reaction the polyacrylic gels, we assume the opposite situation: the rate of the MM reaction is larger than that of the CM reaction. Consequently the polymer is formed first and the process of crosslinking takes place slowly.

One consequence of our assumption regarding the different formation of the gels is that in the case of polyacrylic gels, the fluctuations in the crosslink density will be small and the elastic modulus can be seen as the average of the moduli in the different regions of the gel. We shall try to explain their elastic behavior by the influence of the entanglements.

However, in the case of the polyacrylamide gels, the crosslink density fluctuations are so strong that it is not possible to consider the elastic modulus as merely the average of the local moduli A more appropriate approach is necessary. On the other hand, it is clear that the entanglement contribution (which clearly exists in these gels) cannot explain the saturation or the maximum of $G(c)$.

3.1. Polyacrylamide Gels. - The effective elastic modulus $G_{\mathrm{e}}$ is that of a composite medium made of stiff inclusions (elastic modulus $G_{1}$, relative volume $v$ ) and a soft matrix (elastic modulus $G_{\mathrm{m}}$, relative volume 1-v). We shall denote $p_{1}\left(p_{\mathrm{m}}\right)$ and $c_{\mathrm{s}}\left(c_{\mathrm{m}}\right)$ the polymers and crosslink concentrations in the inclusions (matrix) and we have $p_{1}>p_{\mathrm{m}}$ and $c_{1}>c_{\mathrm{m}}$.

We can understand the variation of $G$ with $c$ by a competition between two mechanisms: one giving an increase of $G$ and the other a decrease, when $c$ increases. One of the possible pictures is the following. We can make a reasonable assumption that the depletion process of the matrix (giving $c_{\mathrm{m}}<c<c_{1}$ and $p_{\mathrm{m}}<p<p_{1}$ ) will be stronger and stronger when 
$c$ increases. Consequently, $G_{\mathrm{m}}$ will be a decreasing function of $c\left(\mathrm{~d} G_{\mathrm{m}} / \mathrm{d} c<0\right)$ and this is the first mechanism for the decrease of $G$ when $c$ increases. At the same time, there is an enrichment of the inclusion in crosslink molecules and polymer giving an elastic modulus of the inclusion which becomes larger and larger when $c$ increases $\left(\mathrm{d} G_{\mathrm{i}} / \mathrm{d} c>0\right)$. It is the second mechanism which gives an increase of $G_{\mathrm{e}}$.

To be more specific, we shall use the result of the theory of composite materials [14]. For an incompressible material like the gels, the effective elastic modulus $G_{e}$ is given by

$$
G_{\mathrm{e}}=G_{\mathrm{m}}\left[1+\frac{v\left(G_{1}-G_{\mathrm{m}}\right)}{G_{\mathrm{m}}+4(1-v)\left(G_{1}-G_{\mathrm{m}}\right)}\right]
$$

It is easy to verify that $G_{\mathrm{e}}$ is an increasing function of $G_{\mathrm{m}}, G_{1}$ and $v$.

At low $c$, it is very likely that $G_{1}$ and $G_{m}$ are not too different and (2) reduces to

$$
G_{\mathrm{e}}=v G_{1}+(1-v) G_{\mathrm{m}}
$$

which express that $G_{\mathrm{e}}$ is the average of the different elastic moduli $G_{\mathrm{i}}$ and $G_{\mathrm{m}}$. In such a case $G_{\mathrm{e}}$ increases with $c$ and if the chain interaction is weak, the dependence of $G_{\mathrm{e}}$ with $c$ is linear, as experimentally observed. But for larger $c$, one has $G_{1} \gg G_{\mathrm{m}}$ (strong fluctuations regime) and the complete expression (2) must be used, when the competition between the two mechanisms takes place.

From the results already published, it is found that $\left(\mathrm{d} G_{\mathrm{m}} / \mathrm{d} c\right)<0[15]$ and that $\left(\mathrm{d} G_{1} / \mathrm{d} c\right)>0$, in accordance with our analysis. It was also found that $(\mathrm{d} v / \mathrm{d} c)<0[10,12]$, increasing the tendency of $G_{\mathrm{e}}$ to decrease when $c$ increases.

The application of the precedent ideas to the polyacrylamide gels are only tentative and needs a confirmation from a quantitative comparison. It is possible to show from (10) that the inclusions will modify significantly $G_{\mathrm{e}}$ only if the difference $G_{1}-G_{\mathrm{m}}$ is large enough and $v$ not too small. At the present stage, we do not have reliable values of the relevant quantities for ascertaining the validity of the model.

The model we proposed can be correct only if-the stiff clusters are well separated. When the polymer content increases, percolation of dense regions will occur and another model is needed. Above the percolation threshold, the elastic modulus is controlled by the dense phase [16] and not by the soft matrix. In such a situation, one expects a regular increase of $G$ with $c$ as observed for samples with $p>20 \%$. This value of $p$ can be seen as the percolation threshold in this system. Effectively, in three dimensions, the threshold for a disordered system is near $20 \%$ [17]. This is to be connected to the finding of reference [12] regarding the tendency of the system to be homogeneous for $p>16 \%$.

To close this section, we mention the prediction of Vilgis [4] about inhomogeneous gels. Vilgis predicts first that the intensity of neutron scattering at small angle will be modified by the inhomogeneities and this was verified experimentally [7]. The second prediction is that the elastic modulus will be that corresponding to the mean values of of $c$ and $p$. This is not in agreement with our experiments in which we found that the elastic modulus saturates or has a maximum when $c$ increases.

3.2. Polyacrylic Gels. - We assumed above that these gels are homogeneous and we propose to include explicitly the entanglements to explain their elasticity. First we calculate $G_{1}$ from equation (1) with $C_{0}=1 / 2$. For $c=0.1 \%$ we find $G_{1}=0.75 \times 10^{5} \mathrm{CGS}$, which corresponds to a Young modulus of $E=2.25 \times 10^{5} \mathrm{CGS}$. This value is slightly larger than the measured value for the softest gel (with the smallest $p$ ). Since $G_{1}$ can be seen as a lower 
bound for a gel, it seems that this gel is almost free of entanglements. Furthermore it is the only example of gel with a linear dependence with $c$ as predicted by (1). All the deviations from the straight line of the $7.9 \%$ polymer gel are supposed to be due to entanglements.

The slipping model of the entanglements [4] takes into account a certain amount of freedom for the entanglements by means of a parameter $\eta$. For $\eta \rightarrow 0$, the entanglements are supposed to be fixed and act as chemical crosslinks. When $\eta$ increases, the entanglements are less effective. The formula for $G$ (in our notation) is

$$
G=\frac{1}{2} k T\left[c+\frac{c_{\mathrm{s}}}{(1+\eta)^{2}}\right]
$$

where $c_{\mathbf{s}}$ is the entanglement concentration. An equivalent expression for $G$ is

$$
G=\frac{1}{2} k T c\left[1+\frac{2 N \alpha}{\left(1+\alpha^{3} N\right)^{2}}\right]
$$

Here $\alpha$ is the ratio between the mean distance between entanglements and the length of the statistical segment. $N$ is the number of the Kuhn segments in a chain. Since $\alpha$ is estimated by Vilgis to be of the order of $10^{-1}$, and in our gels $N \sim 10^{2}, G$ can be written as

$$
G=\frac{1}{2} k T c(1+2 \alpha N)
$$

To compare with the experiment, it is useful to have an expression which includes explicitly the polymer density. First we have $p=2 N c$ (the Kuhn statistical element is about 1.5 the monomer length [12] and we take it equal to 1 by simplicity). Taking into account that, following Vilgis $\eta=\alpha^{3} N$ is a constant of the model we get:

$$
G=\frac{1}{2} k T\left(c+2^{1 / 3} \eta^{1 / 3} c^{1 / 3} p^{2 / 3}\right)
$$

Qualitatively, this expression gives a correct description of the elastic behavior of the polyacrylic gels. $G$ is an increasing function of $c$ and $p$, as observed. If $p$ is small the contribution due to the entanglements may be negligible and when $p$ increases this contribution may be the strongest. From our results we can even get an order of magnitude for $\eta$ in writing the two following conditions a) for $p=7.9 \%$ the "normal" contribution $\left(G_{1}\right)$ is larger than the entanglement contribution $\left(G_{2}\right)$. b) for $p=14 \%$ we have the inverse situation: $G_{1}<G_{2}$. We found that $\eta$ must be between 0.18 and 0.13 which are very reasonable values. However, for a quantitative comparison the expression (8) is not adapted to our results. The functional dependence of $G$ with $c$ or with $p$ cannot be described by (8). The conclusion of this analysis are in fact in agreement with Vilgis which does not claim to provide accurate relationships but only qualitative estimates.

\section{Conclusion}

The elastic behavior of the polyacrylamide and polyacrylic gels is completely different. We relate that to the conditions of their formation. We propose to analyze the results by assuming two different kinds of defects: inhomogeneities in the polyacrylamide gels and entanglements in the polyacrylic gels. We tried to analyze the results following these assumptions but it was not possible to get a complete satisfactorily picture. In the case of the polyacrylamide, we feel that there is a need to get a precise determination of the microstructure of these gels. For the polyacrylic gels, the slipping model of the entanglements gives a good qualitative agreement with the experimental results. 


\section{Acknowledgments}

The author thanks Prof. V. Oshmian and Dr. Y. Cohen for very useful discussions and P. Ron for his technical help. This research was supported by the fund for the promotion of research at the Technion, and by the Technion V.P.R. Fund - B. and G. Greenberg Research Fund (Ottawa).

\section{References}

[1] J E. Mark and E. Erman Eds., Elastomeric Polymer Network (Prentice Hall, Englewood Cliffs, 1992).

[2] Schosseler F, Ilmain F. and Candau S.J., Macromolecules 24 (1991) 225

[3] Cohen Y., Ramon O., Kopelman I J. and Mizrahi S., J Polym Scr. Polym. Phys 30 (1992) 1055

[4] Vilgis T.A., Reference [ [1], Ch 5, p 32.

[5] Dusek K and Prins W, Adv Polym. Scı. 6 (1969) 1.

[6] Barrat J.L., Joanny J.F. and Pincus P, J Phys. II France 2 (1992) 1531.

[7] Mendes E, Jr, Linder P., Buzier M., Boue F. and Bastide J, Phys Rev Lett. 66 (1991) 1595.

[8] Mendes E., Jr , Girard B , Picot C , Buzier M., Boue F and Bastide J , Macromolecules 26 (1993) 6873.

[9] Bastide J., Leibler L. and Prost J., Macromolecules 23 (1990) 1821.

[10] Malpamace F., Micalı N, Vasi C., Bansıl R., Pajevic S and Sciortıno F., J. Phys. II France 2 (1992) 2081.

[11] Tsong-Pin Hsu, Dong Sung Ma and Cohen C, Polymer 24 (1983) 1273.

[12] Wess N., van Vliet T. and Silberberg J., J. Polym Scr Polym Phys. 17 (1979) 2229.

[13] Baselgo J., Hernandez-Fuentes I, Pierola I F and Llorente M.A , Macromolecules 20 (1987) 3060; 21 (1988) 2594; 22 (1989) 3356.

[14] Hashin Z., $J$ Appl. Mech. 50 (1983) 481

[15] Hecht A M , Duplessix R. and Geissler E., Macromolecules 18 (1985) 2167.

[16] Gai M.I., Manevich L.I. and Oshmian V G., Sov. Phys. Dokl. 29 (1984) 505.

[17] Stauffer D., Phys. Rep. 54 (1979) 1. 